\title{
Pulmonary Sarcoidosis with Usual Interstitial Pneumonia Distributed Predominantly in the Lower Lung Fields
}

\author{
Kouichi Nobata ${ }^{1}$, Takahiko Kasai ${ }^{2}$, Masaki Fujimura ${ }^{3}$, Masayuki Mizuguchi ${ }^{1}$, \\ Kouichi Nishi ${ }^{1}$, Yoshihisa Ishiura ${ }^{3}$, Masahide Yasui $^{3}$ and Shinji Nakao ${ }^{3}$
}

\begin{abstract}
Nodular and reticular opacities were detected in both lower lung fields of a 75-year-old man in 2000. Bronchoscopy revealed pulmonary sarcoidosis. In 2002, nodular and reticular opacities were shown in the right upper lobe, and video-assisted thoracoscopic surgery was performed. The histological findings revealed usual interstitial pneumonia (UIP)-like lesions, whereas non-caseous granulomas were not detected. In the present case of pulmonary sarcoidosis, nodular and reticular opacities were predominantly distributed in both lower lung fields, and the histological findings obtained by video-assisted thoracoscopic surgery showed UIPlike lesions. These findings may enlighten the assist in understanding of the process of development of pulmonary sarcoidosis.
\end{abstract}

Key words: pulmonary sarcoidosis, usual interstitial pneumonia, lower lung field

(DOI: 10.2169/internalmedicine.45.1385)

\section{Introduction}

Sarcoidosis is a systemic granulomatous disease of unknown cause. Non-caseating epithelioid cell granuloma is one of its pathological characteristics (1). Although lesions of pulmonary sarcoidosis are often distributed predominantly in the upper lung fields (2), lesions of usual interstitial pneumonia (UIP) are often distributed predominantly in the lower lung fields (3). The pathological findings of interstitial pneumonia do not include non-caseating epithelioid cell granuloma (4). In the advanced stage of the present case, inflammatory fibroid lesions were distributed in the peribronchial zone, which was the feature of sarcoidosis (5), but non-caseating epithelioid cell granulomas were not found. On the other hand, fibroblastic foci were shown in the same specimens, which was the feature of $\operatorname{UIP}(6,7)$, whereas honeycomb lung formation was not found in the computed tomographic (CT) scan. Considering the above findings, this patient's histopathological diagnosis should be 'UIP-like' rather than typical 'UIP'. We describe a patient with pulmonary sarcoidosis distributed predominantly in the lower lung fields and his histopathological features of UIP-like lesions in areas with advanced disease.

\section{Case Report}

Our patient is a 75-year-old former clerk, ex-smoker, without a history of keeping pets. He had been using a down quilt on his bed. In July 2000, a chest radiograph and CT scan taken in a medical check-up showed granular and reticular shadows in both lower lung fields (Fig. 1A, B). Bronchoalveolar lavage (BAL) was performed in the right $\mathrm{B}^{5}$, and transbronchial lung biopsy (TBLB) in the right $\mathrm{S}^{8}$. The recovery rate of bronchoalveolar lavage fluid (BALF) was $47 \%$, and the total number of cells in the fluid was $6.0 \times 10^{5} / \mathrm{ml}$. Cell fractionation showed that the percentages of macrophages, lymphocytes, neutrophils, and eosinophils were $26 \%, 59 \%, 15 \%$, and $0 \%$, respectively. The CD4/CD8 ratio was 10.3 . Biopsy specimens showed that non-caseating epithelioid cell granulomas with a relatively sharp circumference and mild lymphocyte infiltration were seen in the bronchiolar wall (Fig. 2). The level of serum angiotensin converting enzyme (ACE) and lysozyme were as high as

\footnotetext{
${ }^{1}$ Department of Internal Medicine, Ishikawa Prefectural Central Hospital, Kanazawa, ${ }^{2}$ Department of Pathology, Kurobe City Hospital, Kurobe and ${ }^{3}$ Respiratory Medicine, Cellular Transplantation Biology, Kanazawa University Graduate School of Medicine, Kanazawa Received for publication April 19, 2005; Accepted for publication October 13, 2005

Correspondence to Kouichi Nobata, Respiratory Medicine, Cellular Transplantation Biology, Kanazawa University Graduate School of Medicine, 13-1 Takara-machi, Kanazawa 920-8641
} 


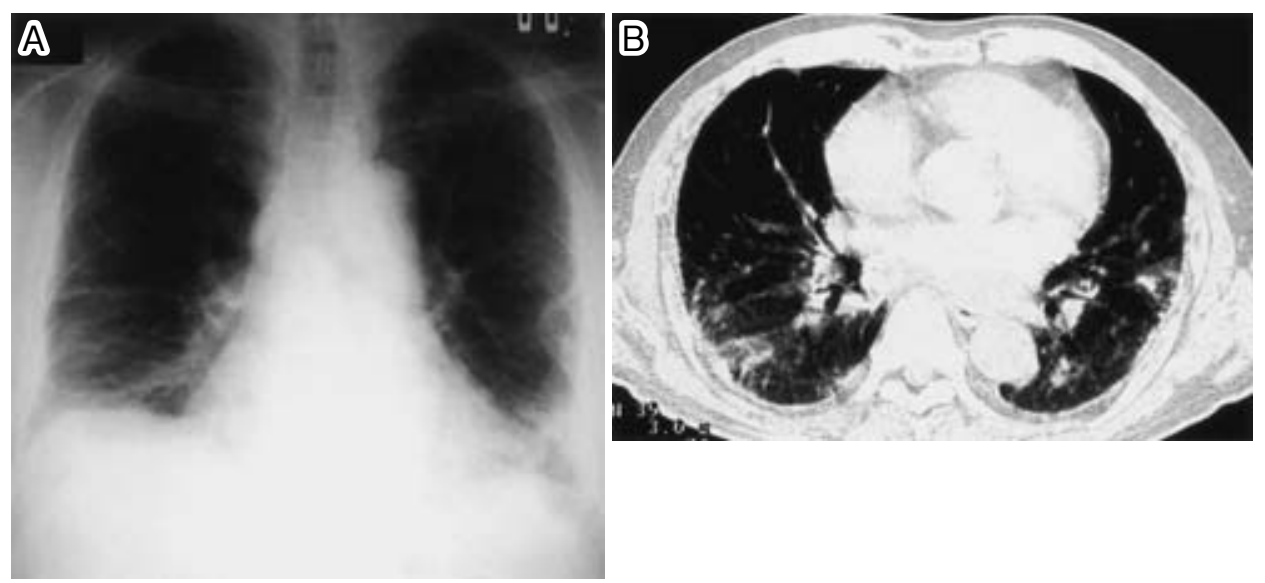

Figure 1. Chest radiograph (A) and CT scan (B) of the lung in July 2000, showing nodular and reticular opacities in the lung fields.

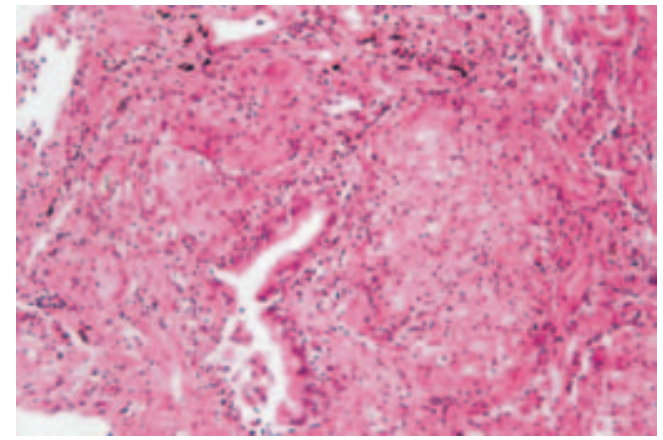

Figure 2. Microscopic finding of lung tissue biopsied from the right lower lobe, showing non-caseous granulomas (HE stain, $\times 200)$.

38.7 IU/l (normal value: $8.3-21.4 \mathrm{IU} / \mathrm{l})$ and $23.8 \mu \mathrm{g} / \mathrm{ml}$ (normal value: $5.0-10.2 \mu \mathrm{g} / \mathrm{ml}$ ), respectively. From the results, pulmonary sarcoidosis was diagnosed. Thallium scintigraphy showed multiple defective images in the septum to the anterior wall, inferior wall, and posterior wall. Electrocardiography showed a complete right bundle branch block, and echocardiography showed a thickened ventricular septum and mild systolic and diastolic dysfunction. Although myocardial biopsy was not performed, these findings showed that there was a possibility of the complication of cardiac sarcoidosis. Therefore, steroid therapy was started $(20 \mathrm{mg} /$ day of prednisolone). After administering prednisolone, the ACE and lysozyme levels were significantly decreased (12.2 $\mathrm{IU} / 1$ and $6.9 \mu \mathrm{g} / \mathrm{ml}$, respectively in February 2002), but the KL-6 level was elevated $(2,430 \mathrm{U} / \mathrm{ml}$ in July $2000,5,470 \mathrm{U} /$ $\mathrm{ml}$ in February 2002).

In April 2002, the patient began to have dyspnea on exertion (grade II, Hugh-Jones classification), and was admitted to our hospital. Although the KL-6 level on admission was as high as $6,828 \mathrm{U} / \mathrm{ml}$, the ACE and lysozyme levels were within normal limits (9.1 IU/l and $6.0 \mu \mathrm{g} / \mathrm{ml}$, respectively). Chest radiograph and CT scan findings revealed granular and reticular shadows in the upper and lower lung fields, but honeycomb lung formation was not found (Fig. 3A, B). BAL was performed in the right $\mathrm{B}^{5}$, and TBLB in the right $\mathrm{S}^{2}$. The recovery rate of BALF was $33 \%$, and the total number of cells in the fluid was $1.0 \times 10^{5} / \mathrm{ml}$. Cell fractionation showed that the percentages of macrophages, lymphocytes, neutrophils, and eosinophils were $13 \%, 68 \%, 19 \%$, and $0 \%$, respectively. $\mathrm{CD} 4 / \mathrm{CD} 8$ ratio was 16.3 . Unfortunately, the specimen obtained by TBLB was not sufficient for analysis, and thoracoscopic lung biopsy from the right $S^{2}$ was performed. The specimens showed that non-caseating epithelioid cell granulomas were not found and inflammatory fibroid lesions were distributed mainly in the bronchioles, pleural membranes, and interlobular septum (Fig. 4A), which were accompanied by swelling of type-II alveolar epithelium and fibroblastic foci (Fig. 4B). It was difficult to differentiate pulmonary sarcoidosis from UIP, because inflammatory fibroid lesions were distributed and inflammatory lesions of different phases were mixed in.

After the thoracoscopic lung biopsy was performed, hypoxaemia was progressive and radiographic findings were worsening. At this point, high dose steroid therapy was started (pulse therapy and $60 \mathrm{mg} /$ day of prednisolone). After the treatment, the patient became better and his KL-6 level gradually decreased. On the other hand, the ACE and lysozyme levels remained within normal limits without any remarkable changes. He has been kept under observation as an outpatient.

The patient was suspected to have chronic hypersensitivity pneumonitis because of his history of using a down quilt on the bed. Thus, specific antibodies to excretory substances from doves and fungi in conserved serum were measured; only the antibody to Trichosporon mucoides was slightly positive, with an antibody level of 0.466 (normal value: 0.3 or lower). However, as it may become slightly positive even after non-specific exposure, we could not make a definite diagnosis of chronic hypersensitivity pneumonitis. He has not shown any recurrence of symptoms or worsening of radio- 

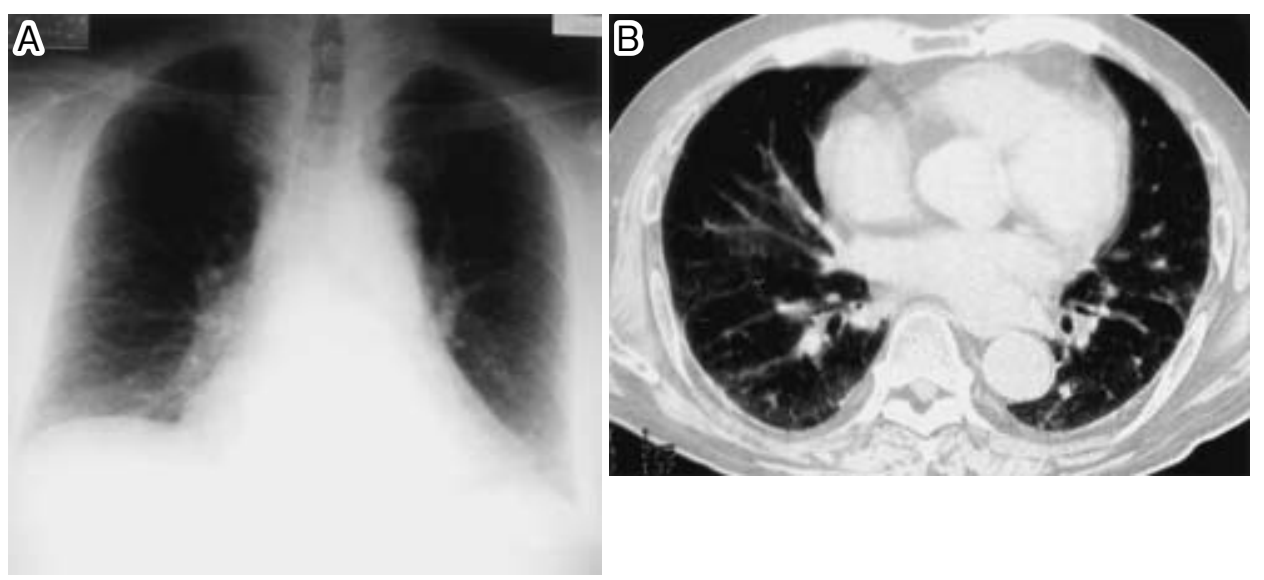

Figure 3. Chest radiograph (A) and CT scan (B) of the lung in June 2002, showing nodular and reticular opacities in the lung fields. Honeycomb lung formation was not found.
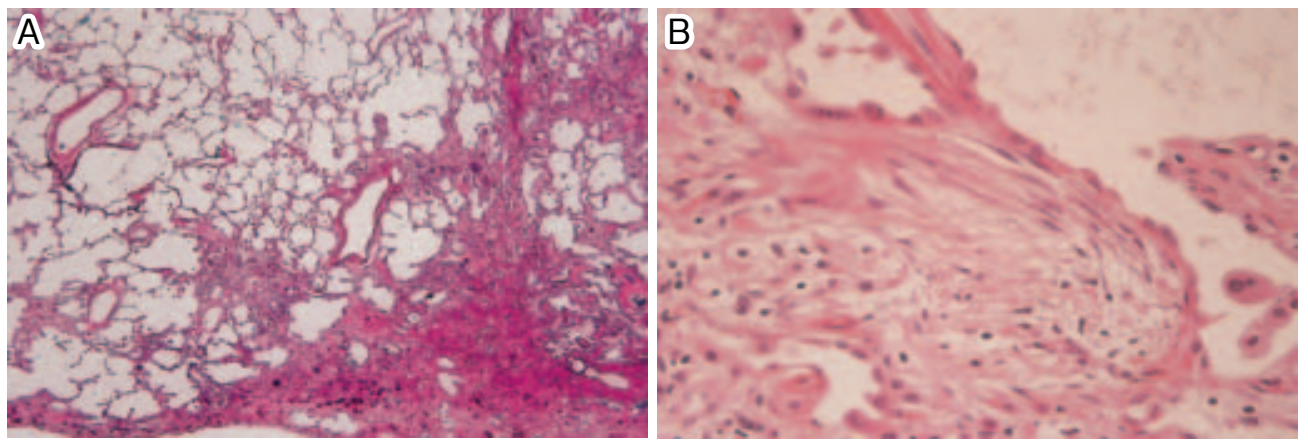

Figure 4. Microscopic finding of lung tissue biopsied from the right upper lobe, showing peripheral and peribronchiolar fibrosis (A) and fibroblastic foci (B), which are pathologic findings of a usual interstitial pneumonia-like lesion, without non-caseous granulomas (HE stain, $\times 200$ ).

graphic findings since he discharged from the hospital. Therefore, from a clinical point of view, we do not think that he had developed chronic hypersensitivity pneumonitis.

There is a possibility that pulmonary sarcoidosis complicated idiopathic pulmonary fibrosis (IPF) during the observation period. However, it is difficult to identify whether it is pulmonary sarcoidosis with IPF or the fibrosis stage of pulmonary sarcoidosis itself.

\section{Discussion}

Here we described a case of pulmonary sarcoidosis that predominantly developed in the lower lung fields and then showed histopathological features of UIP-like lesions in areas with the advanced disease. It is noteworthy that fibrosis and deflation arose in the lower lung fields, not in the upper lung fields, and that there were histopathological features of UIP-like lesions.

Imaging findings in a case of pulmonary sarcoidosis usually include small nodules that are distributed over wide areas in the bundle of blood vessels in the bronchial tube and pleural membranes, thickening of the interlobular septum, distortion of the structure, and concentration of nodular shadows (8). On the other hand, honeycomb lung, cyst for- mation and bronchiectasis, and pulmonary fibrosis are rarely reported (8). Although lesions of sarcoidosis in the lung fields can arise in any areas, they are often predominantly distributed in the upper lung fields as compared to other diffuse diseases of the lungs. In particular, cases of fibrotic pulmonary sarcoidosis have been reported to involve deflation of the upper lobe (2). In the present case, however, the lesions arose from the lower lobe, therefore, this is an atypical case of pulmonary sarcoidosis.

Nicholson et al (6), and Flaherty et al (7) reported that a diagnosis of interstitial pneumonia could be established by fibroblastic foci. In the present case, non-caseating epithelioid cell granulomas were found in pathologic specimens obtained by TBLB in 2000, which was thought to be peculiar to sarcoidosis. In the final stage of typical pulmonary sarcoidosis, narrowing and occlusion of the bronchioles occur mainly in the upper lobe $(9,10)$, which may be caused by fibrosis of granulomatous lesions that are distributed around the bronchiole. In the present case in 2002, noncaseating epithelioid cell granuloma was not observed, and fibrosis was seen around the bronchioles. There were not only features of the final stage of pulmonary sarcoidosis, but also those of UIP such as fibroblastic foci and different phases of inflammatory processes. Thus, this case seemed to 
be different from the typical pulmonary sarcoidosis in the final stage.

It is difficult to clarify the reason why the level of lymphocytes was high in the BALF in 2002 but the ACE and lysozyme levels were low. Hypersensitivity pneumonitis might not be complicated and non-caseating epithelioid cell granuloma had disappeared. It is suspected that steroid therapy decreased the ACE and lysozyme levels with disappearing non-caseating epithelioid cell granuloma, but the activity of sarcoidosis still remained.

The KL-6 level showed a change regardless of the steroid therapy, and became higher when the shadows in the right upper lobe developed. However, KL-6 has also been reported to be elevated in cases of pulmonary sarcoidosis (11), and sarcoidosis is a disease that can cause mononuclear cell infiltration in the alveolar septa, in other words, alveolitis $(12,13)$, a granulomatous disease, and also an interstitial pulmonary disease. Therefore, it is presumed that the change in the KL-6 level in this patient was caused by swelling and growth of type-II alveolar epithelium as seen in thoracoscopic lung biopsy specimens and modification of pulmonary tissues such as fibroblastic foci. It is difficult to pathologically determine whether the change was caused by the fibrosis stage of pulmonary sarcoidosis or the presence of another disease that could lead to an interstitial change. Sarno et al (9) reported that the final stage of pulmonary sarcoidosis might show features similar to those seen in the final stage of UIP. According to the comment of Dr. Take- mura of the Nihon Red Cross Medical Center, non-caseating epithelioid cell granulomas were shown in the TBLB specimens in 2000, which was thought to be pulmonary sarcoidosis, but there was no view of interstitial pneumonia such as hyperplasia of alveolar epithelium. Regarding the VATS specimens in 2002, granulomas were not detected, but fibrosis distributed along bronchovascular bandles was found, which was one of the characteristics of the fibrosis stage of pulmonary sarcoidosis. Fibroblastic foci were found in the specimens in 2002, but they were not so comparable with a typical UIP. Therefore, it is more reasonable to think in a comprehensive manner that, in this patient, the final stage of pulmonary sarcoidosis presented the histopathological observations of UIP-like lesions, rather than to think that the patient's disease was complicated by typical UIP or IPF. It is rare that UIP-like lesions are predominantly distributed in the lower lung fields in a case of pulmonary sarcoidosis (14). Therefore, this case may be valuable to understand the pathologic background of pulmonary sarcoidosis.

The authors are grateful to Dr. Tamiko Takemura (Nihon Red Cross Medical Center) for her collaboration in this work.

Abbreviations: ACE; angiotensin converting enzyme, BAL; bronchoalveolar lavage, BALF; bronchoalveolar lavage fluid, IPF; idiopathic pulmonary fibrosis, TBLB; transbronchial lung biopsy, UIP; usual interstitial pneumonia

\section{References}

1. Statement on sarcoidosis. Joint Statement of the American Thoracic Society (ATS), the European Respiratory Society (ERS) and the Association of Sarcoidosis and Other Granulomatous Disorders (WASOG) adopted by the ATS Board of Directors and by the ERS Exective Committee, February 1999. Am J Respir Crit Care Med 160: 736-755, 1999.

2. Hiraga Y. Case of sarcoidosis initiated by a pulmonary lesion (retrospective study of the clinical development of sarcoidosis). Saishin Igaku 12: 2589-2594, 1969 (in Japanese).

3. Staples CA, Muller NL, Vedal S, Abboud R, Ostrow D, Miller RR. Usual interstitial pneumonia: correlation of CT with clinical, functional, and radiologic findings. Radiology 162: 377-381, 1987.

4. Katzenstein AL, Myers JL. Idiopathic pulmonary fibrosis: clinical relevance of pathologic classification. Am J Respir Crit Care Med 157: 1301-1315, 1998.

5. Padley SP, Padhani AR, Nicholson A, Hansell DM. Pulmonary sarcoidosis mimicking cryptogenic fibrosing alveolitis on CT. Clin Radiol 51: 807-810, 1996.

6. Nicholson AG, Fulford LG, Colby TV, du Bois RM, Hansell DM, Wells AU. The relationship between individual histologic features and disease progression in idiopathic pulmonary fibrosis. Am J Respir Crit Care Med 166: 173-177, 2002.

7. Flaherty KR, Colby TV, Travis WD, Toews GB, Mumford J, Murray S, Thannickal VJ, Kazerooni EA, Gross BH, Lynch JP 3rd,
Martinez FJ. Fibroblastic foci in usual interstitial pneumonia. Am J Respir Crit Care Med 167: 1410-1415, 2003.

8. Wells A. High resolution computed tomography in sarcoidosis: a clinical perspective. Sarcoidosis Vasc Diffuse Lung Dis 15: 140146, 1998.

9. Sarno M, Hasleton PS, Spiteri MA. Sarcoidosis. Spencer's Pathology of the Lung 5th ed., Manchester: 1996: 507-535.

10. Moller DR. Pulmonary fibrosis of sarcoidosis: New approaches, old ideas. Am J Respir Cell Mol Biol 29: S37-S41, 2003.

11. Kobayashi J, Kitamura S. Serum KL-6 for the evaluation of active pneumonitis in pulmonary sarcoidosis. Chest 109: 1276-1282, 1996.

12. Berlin M, Lundahl J, Skold CM, Grunewald J, Eklund A. The lymphocytic alveolitis in sarcoidosis is associated with increased amounts of soluble and cell-bound adhesion molecules in bronchoalveolar lavage fluid and serum. J Intern Med 244: 333-340, 1998.

13. Gal AA, Koss MN. The pathology of sarcoidosis. Curr Opin Pulm Med 8: 445-451, 2002.

14. Demedts M, Wells AU, Anto JM, Costabel U, Hubbard R, Cullinan P, Slabbynck H, Rizzato G, Poletti V, Verbeken EK, Thomeer MJ, Kokkarinen J, Dalphin JC, Taylor AN. Interstitial lung diseases: an epidemiological overview. Eur Respir J Suppl 32: 2S-16 S, 2001.

(C) 2006 The Japanese Society of Internal Medicine http://www.naika.or.jp/imindex.html 